\title{
BMJ Open Is having asthma associated with an increased risk of dying from cardiovascular disease? A prospective cohort study of 446346 Taiwanese adults
}

\author{
Linn Beate Strand, ${ }^{1}$ Min Kuang Tsai, ${ }^{2,3,4}$ Chi Pang Wen, ${ }^{3,4}$ Shu-Sen Chang, ${ }^{5}$ \\ Ben M Brumpton ${ }^{6,7,8}$
}

To cite: Strand LB, Tsai MK, Wen $\mathrm{CP}$, et al. Is having asthma associated with an increased risk of dying from cardiovascular disease? A prospective cohort study of 446346 Taiwanese adults. BMJ Open 2018;8:e019992. doi:10.1136/ bmjopen-2017-019992

- Prepublication history and additional material for this paper are available online. To view these files, please visit the journal online (http://dx.doi. org/10.1136/bmjopen-2017019992).

Received 6 October 2017 Revised 12 March 2018 Accepted 19 April 2018
Check for updates

For numbered affiliations see end of article.

Correspondence to Dr Chi Pang Wen; cwengood@nhri.org.tw and Dr Shu-Sen Chang; shusenchang@ntu.edu.tw

\section{ABSTRACT}

Objectives A significant proportion of cardiovascular disease (CVD) cannot be explained by well-known risk factors such as high cholesterol, hypertension and diabetes. One potential novel risk factor for CVD is asthma. We aimed to investigate the association between asthma and mortality due to CVD.

Design Prospective cohort study.

Setting A large health check-up programme from 1994 to 2011 in Taipei, Taiwan.

Participants 446346 Taiwanese adults. Each participant answered questions regarding asthma history (yes/no) and current daily use of asthma medications (yes/no). Active asthma was defined as those using current daily medications for asthma.

Outcomes The participants were followed for mortality from CVD, coronary heart disease (CHD) and stroke obtained through linkage to the cause-of-death register until 31 December 2011.

Results We found an increased risk of dying from CVD in individuals with active asthma (adjusted HR (aHR) 1.32, $95 \% \mathrm{Cl} 1.08$ to 1.62). The risk of death from $\mathrm{CHD}$ or stroke was increased in a similar manner (aHR 1.16, 95\% Cl 0.78 to 1.73 and aHR $1.23,95 \% \mathrm{Cl} 0.86$ to 1.74 , respectively) although the HR estimates were less precise than that of CVD. For deaths from CVD, CHD and stroke, we found stronger associations with active asthma than non-active asthma, and for CVD and stroke stronger associations in men than women.

Conclusion Our study suggests that asthma, particularly active asthma, may be associated with adverse cardiovascular consequences.

\section{INTRODUCTION}

An estimated 17 million people die of cardiovascular diseases (CVDs) every year. ${ }^{1}$ Among the main forms of CVD are coronary heart disease (CHD) and stroke. ${ }^{1}$ Some well-known factors that increase the risk of developing CVD are unfavourable cholesterol levels, high blood pressure, diabetes and cigarette smoking. A significant proportion of CVD cannot be explained by these known risk factors, and a high incidence of the diseases
Strengths and limitations of this study

- We had objective measures of lung function that allowed us to exclude possible misclassification of asthma with chronic obstructive pulmonary disease.

- However, there is no gold standard for asthma diagnosis, and asthma was confirmed based on self-report.

- Taiwan has some of the lowest mortality rates from cardiovascular disease (CVD) in the world; our sample was relatively young and this led to a relatively small number of CVD deaths.

makes it important to detect other potentially modifiable risk factors. One novel potential risk factor is asthma. As much as 330 million adults worldwide are estimated to have asthma, ${ }^{2}$ emphasising the potential public health importance of any effect of asthma on the occurrence of CVD. Such an association is plausible as it is suggested that asthma is associated with low-grade systemic inflammation and decline in pulmonary function ${ }^{3}$ which has been linked to an increased risk of CVD later in life. ${ }^{45}$ Despite the plausibility of such an association, only a few studies have investigated the association between asthma and risk of CVD. ${ }^{56}$ For example, Iribarren $e t$ $a t^{6}$ recently reported an $\mathrm{HR}$ of $1.22(95 \%$ CI 1.14 to 1.31 ) for CHD-related death or hospitalisation in women with asthma compared with women without asthma after adjusting for demographic and established CHD risk factors; there was no association in men. To strengthen the existing evidence for a causative association, there is a need for large prospective studies on asthma and the risk of CVD, which can additionally investigate the influence of sex.

Approximately, 5.5 million people die from stroke every year. ${ }^{1}$ Among the survivors, the consequences on physical, cognitive 
and emotional functioning are potentially devastating. Despite the serious consequences of stroke, asthma as a novel risk factor has not been thoroughly investigated. ${ }^{57}$ Our aim was to examine whether asthma at baseline was associated with an increased risk of deaths from CVD, and more specifically, CHD and stroke using a large cohort of more than 400000 Taiwanese adults participating in a health check-up programme.

\section{METHODS}

\section{Study population}

The study cohort consisted of 593225 Taiwanese adults aged 20 years or older who participated in a large health check-up programme from 1994 to 2011, run by MJ Health Management Institution, Taipei, Taiwan (https://www.mjclinic.com.tw). The baseline questionnaire included questions about history of asthma and current use of asthma medications. The participants went through a number of biochemical tests and physical examinations as described previously. ${ }^{8}$ In the present study, $67682(11.4 \%)$ of 593225 adults were excluded due to missing information on asthma or asthma medications, and a further $79197(13.4 \%)$ were excluded as information was missing on potential confounding variables including sociodemographic and life style factors. The analysis included $446346(75 \%)$ participants who attended check-ups between 1998 and 2011 and who had full information on asthma, asthma medications and potential confounding variables.

\section{Asthma}

Participants answered questions related to asthma history (yes/no) and current daily use of asthma medications (yes/no). We first defined asthma as those reporting a history of asthma or current daily use of asthma drugs, and then grouped these individuals into two subgroups: (1) non-active asthma (those who only reported a history of asthma but not current use of asthma medications) and (2) active asthma (those who reported current daily use of asthma medications). Self-reported asthma is commonly used in population-based studies; this approach has been evaluated and displays reasonable sensitivity and specificity. ${ }^{910}$

\section{CVD mortality}

From baseline, the participants were followed for mortality until the end of follow-up, 31 December 2011, through linkage to the Taiwanese cause-of-death register using the national identification numbers. The International Classification of Diseases, 9th Revision (ICD-9) or 10th Revision (ICD-10) codes were used to identify mortality from CVD (ICD-9 390-459; ICD-10 I00-I99), CHD (ICD-9 codes 410-414; ICD-10 I20-I25) and stroke (ICD-9 430-438; ICD-10 I60-I69). A previous study showed good accuracy of cause-of-death coding for both heart diseases and stroke in Taiwan. ${ }^{11}$

\section{Covariates}

At the baseline health check-up, participants were wearing light-weight clothes and were measured barefoot. Their weight (to the nearest $0.1 \mathrm{~kg}$ ) and height (to the nearest $\mathrm{mm}$ ) were measured using an auto-anthropometer, Nakamura KN-5000A (Nakamura, Tokyo, Japan) and their body mass index (BMI) was calculated $\left(\mathrm{kg} / \mathrm{m}^{2}\right)$. After $5 \mathrm{~min}$ seated rest, blood pressure was measured two times, at $10 \mathrm{~min}$ intervals, using a computerised auto-mercury sphygmomanometer (CH-5000, Citizen, Tokyo, Japan). We used the mean of the two measurements in our analysis. Glucose, total cholesterol and triglyceride levels were measured in blood collected after overnight fasting using the Hitachi 7150 auto-analyser (Hitachi, Tokyo, Japan). Respiratory functions were measured using an electronic spirometer (HI-501, HI-701 or HI-801; Chest M.I., Tokyo, Japan).

The participants reported their education (middle school or below, high school, junior college, college or higher), marital status (single, married, divorced or separated, widowed), smoking status (never, former, current), alcohol consumption (no or occasional use, former drinking, regular drinking) and physical activity (inactive, low, moderate or high). They also reported whether they had a history of hypertension, diabetes, heart disease, heart surgery, stroke or whether they were taking medications for hypertension, diabetes or heart diseases. Hypertension was defined as the presence of any of the following: reporting a history of hypertension, taking any hypertensive drugs, a systemic blood pressure $\geq 140 \mathrm{~mm}$ $\mathrm{Hg}$ or a diastolic blood pressure $\geq 90 \mathrm{~mm} \mathrm{Hg}$. Diabetes was defined as the presence of any of the following: reporting a history of diabetes, taking diabetes medications or a fasting blood sugar $\geq 126 \mathrm{mmol} / \mathrm{L}$.

\section{Statistical methods}

We used Cox proportional hazards models to investigate the associations of self-reported asthma with CVD, CHD and stroke mortality, respectively. Time at entry was date of recruitment and time of exit was 31 December 2011, or death if earlier.

We estimated the association between asthma (those answering 'yes' to asthma history or use of asthma medications), and CVD, CHD and stroke mortality adjusting for age and sex (model 1). In a second model, we adjusted for age, sex, education and marital status, smoking status, alcohol consumption, physical activity, hypertension, diabetes, BMI, total cholesterol, triglycerides, history of heart disease/heart surgery/use of heart drugs, and history of stroke (model 2). We also investigated the associations between active and non-active asthma and CVD, CHD and stroke mortality.

We investigated whether the associations differed in men and women, between age groups (above and below 60 years) and by smoking status (non-current smoker vs current smokers) by including interaction terms in the models and conducting subgroup analyses. We reported the $p$ value for interaction by comparing models with and without the interaction terms using a likelihood ratio test. 
We conducted a series of sensitivity analyses to test the robustness of our findings. First, we excluded deaths due to CVD, CHD or stroke occurring in the first 2 years of follow-up, as these deaths may be due to pre-existing CVD and not asthma. We excluded participants with a history of heart disease, heart surgery, use of heart medications and a history of stroke at baseline in a second sensitivity analysis. Lastly, we excluded participants with possible chronic obstructive pulmonary disease (COPD) at baseline. In this analysis, we excluded those who had an $\mathrm{FEV}_{1} /$ FVC ratio (forced expiratory volume in $1 \mathrm{~s}$ divided by forced vital capacity) $<0.7$.

The proportional hazards assumption was examined by plotting Schoenfeld residuals with time and by examining their correlation.

Our study complies with the Declaration of Helsinki.

\section{Patient and public involvement}

This study used data from the MJ Health Management Institution, Taipei, Taiwan and patients were not involved in the design, recruitment or conduct of this study. The outcome measures were not informed by patients' priorities, experience and preferences. The MJ Health Management Institution will disseminate all key findings from this study on its website. Participants were thanked in the acknowledgement section.

\section{RESULTS}

Among the 446346 participants, there were 2945 deaths from CVD, 780 deaths from CHD and 1146 deaths from stroke over the follow-up period. At baseline, 3.34\% $(\mathrm{n}=14917)$ of the participants reported to have asthma; $2.60 \%(\mathrm{n}=11603)$ reported to have a history of asthma and $0.74 \%(\mathrm{n}=3314)$ reported to have active asthma. The characteristics of the participants according to baseline asthma status are presented in table 1 .

In the age and sex-adjusted model (model 1), those with asthma had a 27\% (HR 1.27, 95\% CI 1.09 to 1.48 ) increased risk of GVD (table 2). In the multiadjusted model (model 2), the association attenuated (HR 1.13, $95 \%$ CI 0.97 to 1.31). Using a stricter definition of asthma (active asthma), we found that those that reported any current use of asthma medications had a $32 \%$ (HR 1.32, $95 \%$ CI 1.08 to 1.62) increased risk of dying from CVD in model 2. In contrast, there was no association between non-active asthma and CVD mortality (HR 0.96, 95\% CI 0.77 to 1.62 ).

There was evidence that the association between asthma (grouped as non-active and active asthma) and CVD was stronger in men than in women ( $p$ for interaction $=0.005$ in model 2) (table 3). Men who reported asthma had a 25\% increased risk (HR 1.25, 95\% CI 1.04 to 1.49) of dying from CVD in model 2, those with active asthma had a $63 \%$ increased risk (HR 1.63, 95\% CI 1.30 to 2.04) compared with men without asthma, while men with non-active asthma showed no increased risk (HR $0.90,95 \%$ CI 0.68 to 1.19 ). No associations were found in women. When stratifying by age, we found no appreciable differences (online supplementary table 1).

Table 1 Characteristics of the Participants in the MJ Health Check-up Programme, Taiwan $(n=446346)$

\begin{tabular}{|c|c|c|c|c|}
\hline \multirow[b]{2}{*}{ Characteristic } & \multicolumn{2}{|c|}{$\begin{array}{l}\text { Asthma } \\
(\mathrm{N}=14917)\end{array}$} & \multicolumn{2}{|c|}{$\begin{array}{l}\text { No asthma } \\
(\mathrm{N}=431429)\end{array}$} \\
\hline & $\mathbf{n}$ & (\%) & $\mathbf{n}$ & (\%) \\
\hline Female & 6893 & $(46.2)$ & 220253 & $(51.1)$ \\
\hline College or higher education & 5726 & $(38.4)$ & 143649 & (33.3) \\
\hline Married & 8659 & $(58.0)$ & 281011 & $(65.1)$ \\
\hline Current smoker & 3461 & $(23.2)$ & 98730 & (22.9) \\
\hline Regular alcohol use & 1210 & $(8.1)$ & 29884 & (6.9) \\
\hline Physically inactive & 7462 & $(50.0)$ & 220976 & $(51.2)$ \\
\hline $\begin{array}{l}\text { Hypertension (history of hypertension+hypertensive drug } \\
\text { use+systolic blood pressure } \geq 140 \mathrm{~mm} \text { Hg or diastolic blood pressure } \\
\geq 90 \mathrm{~mm} \mathrm{Hg} \text { ) }\end{array}$ & 3121 & $(20.9)$ & 77972 & $(18.1)$ \\
\hline $\begin{array}{l}\text { Diabetes (history of diabetes+diabetes drug use+fasting blood glucose } \geq 126 \text { ) } \\
\mathrm{mmol} / \mathrm{L} \text { ) }\end{array}$ & 841 & $(5.6)$ & 21274 & $(4.9)$ \\
\hline History of heart disease/heart surgery/use of heart drugs & 1045 & $(7.0)$ & 15044 & $(3.5)$ \\
\hline \multirow[t]{2}{*}{ History of stroke } & 129 & $(0.9)$ & 2050 & $(0.5)$ \\
\hline & Mean & (SD) & Mean & (SD) \\
\hline Age (years) & 41.0 & $(15.6)$ & 40.0 & $(13.4)$ \\
\hline Body mass index $\left(\mathrm{kg} / \mathrm{m}^{2}\right)$ & 23.4 & $(3.8)$ & 23.0 & (3.6) \\
\hline Total cholesterol (mmol/L) & 5.0 & $(1.0)$ & 5.0 & $(1.0)$ \\
\hline Triglycerides (mmol/L) & 3.0 & $(2.4)$ & 3.0 & (2.6) \\
\hline
\end{tabular}




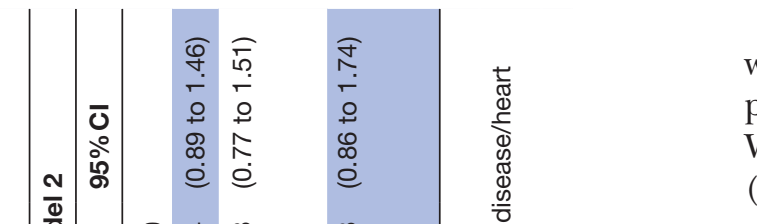

The associations between asthma and CHD mortality were consistent with those of CVD mortality, although less precise due to the smaller number of deaths (table 2). When stratifying by sex, we found a $67 \%$ increased risk (HR 1.67, 95\% CI 1.08 to 2.59) for CHD among men with active asthma in the age-adjusted model (model 1), but the association was largely attenuated when adjusting for additional potential confounders (model 2). We did not find any association among women (table 3 ). We found more than double the risk in those below 60 years (HR $2.57,95 \%$ CI 1.06 to 6.26 ) in model 1 , but the CI was wide and the association was attenuated when adjusting for additional potential confounders (online supplementary table 1).

When investigating mortality due to stroke, we found similar but slightly weaker associations between asthma and stroke mortality than between asthma and CVD mortality (table 2). We found a $77 \%$ increased risk in men with active asthma (HR 1.77, 95\% CI 1.22 to 2.57 ) in model 1, that persisted after adjusting for potential confounders in model 2 (HR 1.62, 95\% CI 1.11 to 2.35) (table 3). This increase was not seen in women ( $p$ for interaction $=0.032$ ). We found no differences in the association between asthma and stroke mortality between age groups (ie, below and above 60 years) (online supplementary table 1 ).

When excluding deaths occurring during the two first years of follow-up, the results did not change considerably for any of the cardiovascular outcomes (online supplementary table 2). When excluding participants with a history of heart disease, heart surgery, use of heart medications and a history of stroke at baseline, the results were generally similar to those of the main analysis except that the association between asthma and CHD was largely attenuated, although the number of CHD deaths was small ( $\mathrm{n}=13$ and 10 in the non-active and active asthma groups, respectively) in this analysis (online supplementary table 3). When excluding individuals with FEV1/FVC ratio $<0.7$ at baseline, the analysis based on the remaining sample showed that the effect estimate remained the same although the CI widened slightly because of lower power in this analysis (online supplementary table 4).

Overall, there was no statistical evidence for a difference in the association between asthma and CVD/CHD/ stroke mortality between current smokers and non-current smokers (all p for interaction $>0.05$ ), although there was a tendency towards stronger associations in current smokers than in non-smokers or ex-smokers (online supplementary table 5).

\section{DISCUSSION}

To the best of our knowledge, this is the largest study to date on asthma and CVD mortality. In this sample of more than 400000 Taiwanese adults, we found an increased risk of dying from CVD in individuals with active, but not non-active, asthma. The risk of death from CHD or stroke was increased in a similar manner, however, in sensitivity 


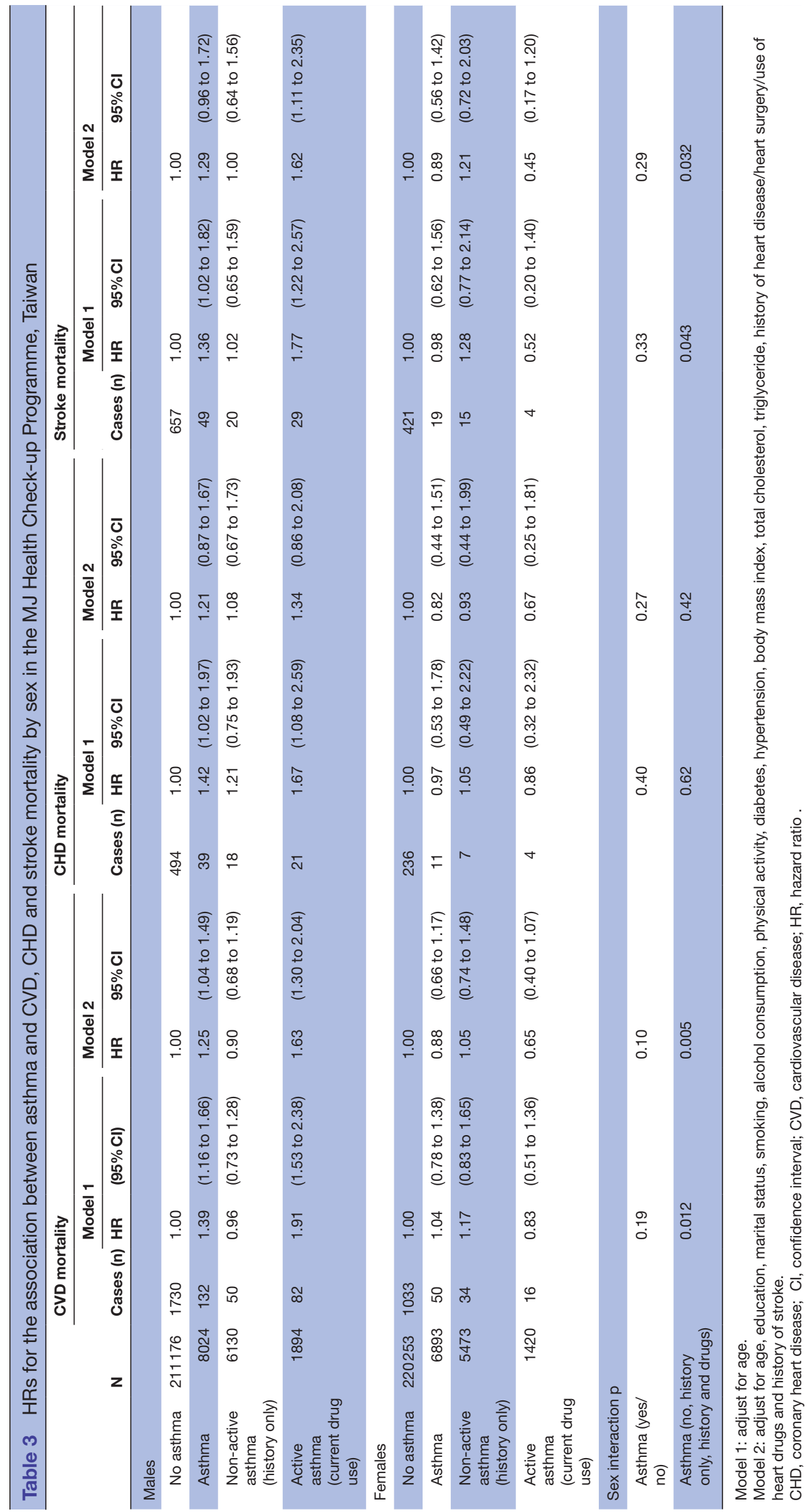


analysis excluding those with previous heart disease, only the associations with CVD and stroke mortality remained. For deaths from CVD and stroke, we found stronger associations with active asthma in men than in women.

A few previous studies have investigated the association between asthma and risk of CVD. One very large recent study including almost one million Italian adults agreed with our findings and reported asthma to be moderately associated with different CVDs. ${ }^{12}$ Additionally, this study did not find any differences in the association between men and women, while our study observed a stronger association in men. This Italian study was cross-sectional, however, and there is no way of knowing whether the CVD diagnosis preceded the asthma diagnosis. Additionally, this study did not have access to spirometry measurements such as $\mathrm{FEV}_{1}$ and FVC and thus was not able to exclude those with a $\mathrm{FEV}_{1} / \mathrm{FVC}$ ratio of less than 0.7 to minimise the possibility of misclassification between asthma and COPD.

A smaller study was undertaken in Australia including approximately 4000 people among whom 500 were classified as having asthma. ${ }^{13}$ Individuals with asthma were identified by self-report, and through identification of significant reversibility of airway obstruction. The results were in accordance with our findings and when subjects with COPD were excluded from the analysis $\left(\mathrm{FEV}_{1} /\right.$ FVC $<0.7$ and $\mathrm{FEV}_{1}<80 \%$ of predicted), the association between asthma and CVD remained.

CHD contributes to a large part of the burden of $\mathrm{CVD}^{14}$ and the risk of complications or even death is high, thus detecting novel risk factors is essential. A few studies have found an increased risk of CHD in patients with asthma. ${ }^{1516}$ However, the results are inconsistent, and a recent study including almost 16000 participants from the Atherosclerosis Risk in Communities (ARIC) Study failed to confirm this increase in risk of CHD in patients with current asthma (HR $0.69,95 \%$ CI 0.46 to 1.05 ). ${ }^{5}$

In the current study, we generally observed stronger results in men than in women in this adult Taiwanese population. By contrast, other studies have previously found stronger associations in women than men. A large prospective study by Iribarren et $a l^{17}$ found that the increased risk of CHD was higher in women with asthma (HR 1.49, 95\% CI 1.43 to 1.56 ) than in men (HR $1.28,95 \%$ CI 1.21 to 1.34 ) ( $\mathrm{p}$ for interaction <0.001). The authors speculate that the increased risk of CHD among people with asthma may be due to chronic inflammation in people with asthma. Supporting this is the fact that women with asthma tend to have more severe disease than men with asthma, ${ }^{18}$ and likely more systemic inflammation.

In spite of the devastating consequences of stroke, asthma as a novel risk factor has not been thoroughly investigated and only a few studies have examined this association. The previously mentioned study using data from the ARIC Study, ${ }^{5}$ found similar results to ours, with a $55 \%$ increased risk of stroke (HR 1.55, 95\% CI 0.95 to 2.52 ) in patients with current asthma compared with those without asthma. A recent meta-analysis of five studies on asthma and stroke found a pooled HR of 1.32 (95\% CI 1.13 to 1.54$).{ }^{19}$ In contrast to our findings, this meta-analysis found that the associations between asthma and stroke were stronger in women (HR 1.42, $95 \%$ CI 1.15 to 1.76 ) than in men (HR $1.19,95 \%$ CI 0.90 to 1.43 ). The authors speculate that sex hormones could play a crucial role in modulating immunological inflammation in asthma. However, our results showed an increased risk in men, but not in women. The underlying reasons driving these differences between studies are not clear and further investigations from countries with different ethnicities may help to shed light on this observation.

The biological mechanisms by which asthma may influence CVD are not known, but several mechanisms have been suggested. Asthma is associated with low-grade systemic inflammation, ${ }^{20}$ which could influence later risk of CVD. ${ }^{21}$ Previous studies also suggest that C-reactive protein is associated with both asthma ${ }^{22}$ and CVD. ${ }^{23}{ }^{24}$ Lung function impairment is also associated with CVD risk. ${ }^{25}{ }^{26}$ Patients with impaired lung function have increased inflammatory markers (including C-reactive protein, interleukin- $1 \beta$ and interleukin- 6$),{ }^{27}$ which are associated with atherosclerosis and cardiovascular events. ${ }^{21}{ }^{28}$ Long-term airway remodelling from the inflammatory response in asthma can produce irreversible airway obstruction and cause a decline in lung function. ${ }^{29}$ In addition, severe asthma exacerbations are associated with a more rapid decline in lung function. ${ }^{30}$ Another hypothesis is that asthma is associated with risk of GVD through asthma medication use, in particular the cardiotoxic effects of beta- $2\left(\beta_{2}\right)$ agonists. ${ }^{3132}$ Finally, asthma may be associated with GVD due to other factors such as obesity, ${ }^{33}$ smoking ${ }^{34}$ or physical inactivity. ${ }^{35}$ It is also possible that the association between asthma and CVD is due to comorbidities such as diabetes which has a strong link to $\mathrm{CVD}^{36}$ and also to asthma. ${ }^{37}$ In our study, we adjusted for these potential confounders and it is unlikely that confounding by these factors would be behind the observed associations.

To the best of our knowledge, our study is the largest to date, investigating the association between asthma and risk of death from CVD. Despite the large study size, the objective measures of lung function that allowed us to exclude possible misclassification of asthma with COPD, and the wide range of potential confounders included in our models, the limitations of this study must be considered.

There is no gold standard for asthma diagnosis and despite using spirometry measures to exclude possible COPD, asthma in this study was indicated by self-report. Additionally, we cannot rule out the potential misdiagnosis of heart disease as asthma. Among those diagnosed with asthma, we were unable to separate patients with asthma by disease onset (ie, childhood vs adulthood) or any other phenotype such as obesity-related, exercise-induced and neutrophilic asthma, ${ }^{38}$ and it is possible that 
these phenotypes differ in regard to asthma triggers, ${ }^{39}$ gender distribution ${ }^{39}$ and systemic inflammation. ${ }^{40}$ For example, obesity-related asthma which usually develops in adulthood might be of particular importance to the development of CVD. However, since we were unable to differentiate between these subgroups our observations may not be representative of each. Finally, patients who used asthma medications less frequently than daily were grouped together with other patients with only a history of asthma, defining a very heterogeneous group which might be a limitation of this study.

Taiwan and some other East Asian countries such as Japan and Hong Kong have some of the lowest mortality rates from CVD in the world. ${ }^{41} 42$ Furthermore, our sample was fairly young (mean age 40.4 years), and these may have contributed to the relatively small number of CVD deaths in our study. This may have reduced our power to detect any small effects of asthma on risk of CVD death.

Observational studies inherently limit causal inference. Although we adjusted for a number of potential confounders in our analyses, there is a possibility of uncontrolled confounding contributing to the observed associations. Specifically, we did not have information on pack-years of cigarette smoking which could be a potential confounder. However, any residual confounding would need to be strongly associated with both asthma and CVD mortality and be unrelated to the covariates included in our models. Additionally, bias due to the exclusion of participants with missing information might have limited our study.

Finally, the sample was from a health check-up programme run by a private company and the participants may have come from a somewhat more advantaged socioeconomic position and therefore our findings may not be representative of the general population. However, the cohort was similar to the general population reported in a national survey ${ }^{43}$ in terms of certain characteristics including the prevalence of smoking.

\section{CONCLUSION}

Our study suggests that asthma, particularly active asthma, may be associated with adverse cardiovascular consequences. For deaths from CVD and stroke, the association was stronger in men than in women. The associations persisted even after adjustment for established CVD risk factors. Further studies are needed to better elucidate the mechanisms underlying this association and to clarify any sex differences in the association.

\footnotetext{
Author affiliations

${ }^{1}$ Department of Public Health and Nursing, Norwegian University of Science and Technology, Trondheim, Norway

${ }^{2}$ Institute of Epidemiology and Preventive Medicine, College of Public Health, National Taiwan University, Taipei City, Taiwan

${ }^{3}$ China Medical University Hospital, Taichung, Taiwan

${ }^{4}$ Institute of Population Health Sciences, National Health Research Institutes, Miaoli County, Taiwan
}

${ }^{5}$ Institute of Health Behaviors and Community Sciences, College of Public Health, National Taiwan University, Taipei City, Taiwan

${ }^{6}$ Department of Thoracic and Occupational Medicine, St. Olavs Hospital, Trondheim, Norway

${ }^{7}$ K.G. Jebsen Center for Genetic Epidemiology, Department of Public Health and Nursing, Norwegian University of Science and Technology, Trondheim, Norway ${ }^{8} \mathrm{MRC}$ Integrative Epidemiology Unit, University of Bristol, Bristol, UK

Acknowledgements We thank the participants. Raw data used for analysis in this research were provided by MJ Health Resource Center (Authorisation code: MJHRFB2014001C). The MJ Health Resource Foundation is responsible for the data distribution. Any interpretation or conclusions drawn from the research analysis do not represent the views of MJ Health Resource Center.

Contributors LBS wrote the analysis plan and wrote the first draft of the manuscript. MKT did the data analysis. C-PW supervised the work and reviewed the manuscript. S-SC helped supervise the work, reviewed the manuscript and coordinated the collaboration between the researchers. BMB designed the study, helped write the analysis plan, wrote the methods section of the manuscript and reviewed the manuscript. All authors confirmed that they reviewed and approved the final version of the manuscript.

Funding This work was supported by funding for the Liaison Committee between the Central Norway Regional Health Authority and the Norwegian University of Science and Technology awarded to LBS and BMB. BMB works in a research unit funded by Stiftelsen Kristian Gerhard Jebsen; Faculty of Medicine and Health Sciences, NTNU; The Liaison Committee for education, research and innovation in Central Norway and the Joint Research Committee between St. Olavs Hospital and the Faculty of Medicine and Health Sciences, NTNU.

Competing interests None declared.

Patient consent Obtained.

Ethics approval The China Medical University Hospital Ethics Committee has approved the research protocol. The study was approved by the National Health Research Institutes, Taiwan and the MJ Health Management Institution.

Provenance and peer review Not commissioned; externally peer reviewed.

Data sharing statement No additional data are available.

Open Access This is an Open Access article distributed in accordance with the Creative Commons Attribution Non Commercial (CC BY-NC 4.0) license, which permits others to distribute, remix, adapt, build upon this work non-commercially, and license their derivative works on different terms, provided the original work is properly cited and the use is non-commercial. See: http://creativecommons.org/ licenses/by-nc/4.0/

(C) Article author(s) (or their employer(s) unless otherwise stated in the text of the article) 2018. All rights reserved. No commercial use is permitted unless otherwise expressly granted.

\section{REFERENCES}

1. McKay J, Mensah GA, Greenlund K. The atlas of heart disease and stroke: World Health Organization, 2004.

2. Global asthma network. The global asthma report 2014;2014 http:// www.globalasthmareport.org/

3. Wouters EF, Reynaert NL, Dentener MA, et al. Systemic and local inflammation in asthma and chronic obstructive pulmonary disease: is there a connection? Proc Am Thorac Soc 2009;6:638-47.

4. Koenig W, Sund M, Fröhlich M, et al. C-Reactive protein, a sensitive marker of inflammation, predicts future risk of coronary heart disease in initially healthy middle-aged men: results from the MONICA (Monitoring Trends and Determinants in Cardiovascular Disease) Augsburg Cohort Study, 1984 to 1992. Circulation 1999;99:237-42

5. Schanen JG, Iribarren C, Shahar E, et al. Asthma and incident cardiovascular disease: the Atherosclerosis Risk in Communities Study. Thorax 2005;60:633-8.

6. Iribarren C, Tolstykh IV, Eisner MD. Are patients with asthma at increased risk of coronary heart disease? Int $\mathrm{J}$ Epidemiol 2004;33:743-8.

7. Onufrak SJ, Abramson JL, Austin HD, et al. Relation of adultonset asthma to coronary heart disease and stroke. Am J Cardiol 2008;101:1247-52. 
8. Chang SS, Wen CP, Tsai MK, et al. Adiposity, its related biologic risk factors, and suicide: a cohort study of 542,088 taiwanese adults. Am $J$ Epidemiol 2012;175:804-15.

9. Torén K, Brisman J, Järvholm B. Asthma and asthma-like symptoms in adults assessed by questionnaires. A literature review. Chest 1993;104:600-8.

10. de Marco R, Cerveri I, Bugiani M, et al. An undetected burden of asthma in Italy: the relationship between clinical and epidemiological diagnosis of asthma. Eur Respir J 1998;11:599-605.

11. Lu TH, Lee MC, Chou MC. Accuracy of cause-of-death coding in Taiwan: types of miscoding and effects on mortality statistics. Int $J$ Epidemiol 2000;29:336-43.

12. Cazzola M, Calzetta L, Bettoncelli G, et al. Cardiovascular disease in asthma and COPD: a population-based retrospective cross-sectional study. Respir Med 2012;106:249-56.

13. Appleton SL, Ruffin RE, Wilson DH, et al. Asthma is associated with cardiovascular disease in a representative population sample. Obes Res Clin Pract 2008;2:91-9.

14. Roger VL. Epidemiology of myocardial infarction. Med Clin North Am 2007;91:537-52.

15. Torén K, Lindholm NB. Do patients with severe asthma run an increased risk from ischaemic heart disease? Int $\mathrm{J}$ Epidemiol 1996;25:617-20.

16. Musk AW, Ryan GF, Perera DM, et al. Mortality from asthma in Western Australia. Med J Aust 1987;147:423-7.

17. Iribarren C, Tolstykh IV, Miller MK, et al. Adult asthma and risk of coronary heart disease, cerebrovascular disease, and heart failure: a prospective study of 2 matched cohorts. Am J Epidemiol 2012;176:1014-24. kws181.

18. Becklake MR, Kauffmann F. Gender differences in airway behaviour over the human life span. Thorax 1999;54:1119-38.

19. Wen LY, Ni H, Li KS, et al. Asthma and risk of stroke: A systematic review and meta-analysis. J Stroke Cerebrovasc Dis 2016;25:497-503.

20. Ishmael FT. The inflammatory response in the pathogenesis of asthma. J Am Osteopath Assoc 2011;111:S11-S7.

21. Willerson JT. Inflammation as a Cardiovascular Risk Factor. Circulation 2004;109:II-2-0.

22. Arif AA, Delclos GL, Colmer-Hamood J. Association between asthma, asthma symptoms and C-reactive protein in US adults: data from the National Health and Nutrition Examination Survey, 19992002. Respirology 2007;12:675-82.

23. Kaptoge S, Di Angelantonio E, Lowe G, et al. C-reactive protein concentration and risk of coronary heart disease, stroke, and mortality: an individual participant meta-analysis. Lancet 2010;375:132-40.

24. Liu Y, Wang J, Zhang L, et al. Relationship between C-reactive protein and stroke: a large prospective community based study. PLoS One 2014;9:e107017.

25. Hozawa A, Billings JL, Shahar E, et al. Lung function and ischemic stroke incidence: the Atherosclerosis Risk in Communities study. Chest 2006;130:1642-9.
26. Gulsvik AK, Gulsvik A, Skovlund E, et al. The association between lung function and fatal stroke in a community followed for 4 decades. $J$ Epidemiol Community Health 2012;66:1030-6.

27. Tamagawa E, van Eeden SF. Impaired lung function and risk for stroke: role of the systemic inflammation response? Chest 2006;130:1631-3.

28. Kuo HK, Yen CJ, Chang $\mathrm{CH}$, et al. Relation of C-reactive protein to stroke, cognitive disorders, and depression in the general population systematic review and meta-analysis. Lancet Neurol 2005;4:371-80.

29. James AL, Palmer LJ, Kicic E, et al. Decline in lung function in the Busselton Health Study: the effects of asthma and cigarette smoking. Am J Respir Crit Care Med 2005;171:109-14.

30. O'Byrne PM, Pedersen S, Lamm CJ, et al. Severe exacerbations and decline in lung function in asthma. Am J Respir Crit Care Med 2009;179:19-24.

31. Au DH, Curtis JR, Every NR, et al. Association between inhaled betaagonists and the risk of unstable angina and myocardial infarction. Chest 2002;121:846-51.

32. Au DH, Lemaitre RN, Curtis JR, et al. The risk of myocardial infarction associated with inhaled beta-adrenoceptor agonists. Am J Respir Crit Care Med 2000;161:827-30.

33. Brumpton B, Langhammer A, Romundstad P, et al. General and abdominal obesity and incident asthma in adults: the HUNT study. Eur Respir J 2013;41:323-9.

34. Coogan PF, Castro-Webb N, Yu J, et al. Active and passive smoking and the incidence of asthma in the Black Women's Health Study. Am J Respir Crit Care Med 2015;191:168-76.

35. Eijkemans M, Mommers M, Draaisma JM, et al. Physical activity and asthma: a systematic review and meta-analysis. PLoS One 2012;7:e50775.

36. Howard BV, Cowan LD, Go O, et al. Adverse effects of diabetes on multiple cardiovascular disease risk factors in women. The Strong Heart Study. Diabetes Care 1998;21:1258-65.

37. Ehrlich SF, Quesenberry CP, Van Den Eeden SK, et al. Patients diagnosed with diabetes are at increased risk for asthma, chronic obstructive pulmonary disease, pulmonary fibrosis, and pneumonia but not lung cancer. Diabetes Care 2010;33:55-60.

38. Wenzel SE. Asthma phenotypes: the evolution from clinical to molecular approaches. Nat Med 2012;18:716-25.

39. Wenzel SE. Asthma: defining of the persistent adult phenotypes. Lancet 2006;368:804-13.

40. Olafsdottir IS, Gislason T, Thjodleifsson B, et al. $\mathrm{C}$ reactive protein levels are increased in non-allergic but not allergic asthma: a multicentre epidemiological study. Thorax 2005;60:451-4.

41. Cheng Y, Chen KJ, Wang CJ, et al. Secular trends in coronary heart disease mortality, hospitalization rates, and major cardiovascular risk factors in Taiwan, 1971-2001. Int J Cardiol 2005;100:47-52.

42. Finegold JA, Asaria P, Francis DP. Mortality from ischaemic heart disease by country, region, and age: statistics from World Health Organisation and United Nations. Int J Cardiol 2013;168:934-45.

43. Wen CP, Levy DT, Cheng TY, et al. Smoking behaviour in Taiwan, 2001. Tob Control 2005; $14: \mathrm{i} 51-\mathrm{i} 55$. 